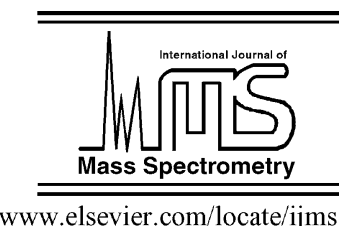

\title{
Temperature dependence of secondary ion emission from tantalum produced by atomic and polyatomic gold projectiles
}

\author{
S.F. Belykh ${ }^{\mathrm{a}, *}$, I.V. Veryovkin ${ }^{\mathrm{b}}$, V.V. Palitsin ${ }^{\mathrm{c}}$, A.V. Samartsev ${ }^{\mathrm{d}}$, \\ A. Adriaens ${ }^{\mathrm{e}}$, F. Adams ${ }^{\mathrm{a}}$ \\ a Department of Chemistry, University of Antwerp (UIA), Universiteitsplein 1, B-2610 Antwerp (Wilrijk), Belgium \\ ${ }^{\mathrm{b}}$ Materials Science Division, Argonne National Laboratory, Argonne, IL 60439, USA \\ ${ }^{\mathrm{c}}$ Department of Physics, Warwick University, CVI 7AL Coventry, UK \\ ${ }^{\mathrm{d}}$ Institute of Experimental Physics, University of Duisburg_Essen, D-45117 Essen, Germany \\ ${ }^{e}$ Department of Analytical Chemistry, Gent University B-9000 Ghent, Belgium
}

Received 12 December 2003; accepted 28 June 2004

linear collision cascades developing in sub-surface regions of solids [1]. While emission mechanisms for neutral atoms and atomic ions produced by atomic projectiles are now well understood [2], the processes of cluster emission [3-6] and charged state formation $[7,8]$ of the emitted clusters remain a subject of ongoing discussions. Yields of both neutral clusters and cluster ions depend on the interaction characteristics in a given "projectile-solid" system defined by the relationship between chemical and physical properties of the projectile 
${ }_{58} \eta^{+}=\frac{Y_{i}\left(M_{n}^{+}\right)}{Y_{n}}$,

and the sample composition. These include, among others, the impact energy and incidence angle of the projectile, the crystalline structure and temperature of the sample, and the surface binding energy (or the heat of sublimation as its first approximation). Variations of these characteristics change the ionized fraction of cluster ions in the total flux of sputtered clusters because of the influence in both cluster sputtering and cluster charge formation processes.

Strong differences in the ionization probability $\eta^{+}$of clusters sputtered by $5 \mathrm{keV} \mathrm{Ar}^{+}$atomic projectiles were reported for two groups of samples (Ag, Ge, In and $\mathrm{Nb}, \mathrm{Ta}$ ) [9]. In this work, the ionization probability is defined as

where $Y_{n}=Y_{i}\left(\mathrm{M}_{n}{ }^{+}\right)+Y_{0}\left(\mathrm{M}_{n}\right) ; Y_{i}\left(\mathrm{M}_{n}{ }^{+}\right)$and $Y_{0}\left(\mathrm{M}_{n}\right)$ are the yields of positively charged $\mathrm{M}_{n}{ }^{+}$and neutral $\mathrm{M}_{n} n$-atomic clusters, respectively. For low melting point elements with low heats of the sublimation such as Ag, Ge, and In, the cluster flux was represented mainly by neutral clusters $\left(\eta^{+}\right.$ $\ll 1)$. However, for high melting point elements with higher heats of sublimation such as $\mathrm{Nb}$ and Ta, $\eta^{+}$quickly increased with $n$ and reached saturation at $\eta^{+}=0.75$ for $\mathrm{Ta}_{5}$ and $\eta^{+}$ $=0.27$ for $\mathrm{Nb}_{7}$, thus demonstrating how significant of the ionized fraction in the cluster emission is for $n>4$.

Non-additive enhancement of sputtering was observed if polyatomic projectiles were used instead of atomic ones [1]. The bombardment of clean surfaces of $\mathrm{Nb}$ and Ta targets with $6 \mathrm{keV} /$ atom $\mathrm{Au}_{x}{ }^{-}$projectiles $(x=1-3)$ caused a non-additive increase of yields of cluster ions $\mathrm{Ta}_{n}{ }^{+}$and $\mathrm{Nb}_{n}{ }^{+}$[10-12], which probably resulted from the non-additive sputtering of neutral clusters rather than from non-additive process of their ionization [13]. This conclusion is in agreement with resent results of Samartsev and Wucher [14]. Using a laser postionization techniques, they found non-additive enhancements in signals of neutral $\operatorname{In}_{n}$ clusters sputtered from an indium target by $5 \mathrm{keV} /$ atom $\mathrm{Au}_{x}{ }^{-}$projectiles $(x=1$ and 2$)$. Based on published results [10-14], it can be stated that, at least for metals bombarded by polyatomic projectiles, non-additive emission enhancement for neutral clusters and cluster ions results from the non-additivity of the sputtering process.

The rise of target temperature $T$ might also affect nonthermal cluster sputtering processes by introducing thermal excitation into the impact region. For $12 \mathrm{keV} \mathrm{Xe}^{+}$sputtering of silver targets, the rise of $T$ in the range of $300-950 \mathrm{~K}$ (when thermal evaporation of the target material is negligible) did not lead to the change in neutral $\mathrm{Ag}_{n}$ cluster yields, while cluster $\mathrm{Ag}_{n}+$ ion yields were enhanced several times [15]. This enhancement was interpreted as the indication of the temperature dependence of the ionization probability for the cluster ion formation process. Generally speaking, for the experiments described by Staudt et al. [15], yields of neutral and ionized clusters should correlate with each other if it is assumed that their sum (the total cluster yield) stays constant. In this case, it seems reasonable to expect that the increase in the cluster ion yield should correlate with the decrease in the yield of neutral clusters. Probably, the change in neutral cluster yields with increasing temperature were not observed for silver targets because of too low intensities of the ionized clusters compared to the neutral ones: $Y_{i}\left(\mathrm{Ag}_{n}{ }^{+}\right) \ll Y_{0}\left(\mathrm{Ag}_{n}\right)$ [15]. In view of these results, it appeared interesting to study temperature dependencies of cluster ion yields in a wide temperature range for other materials, known to have a significant fraction of sputtered clusters ionized, such as tantalum or niobium [9].

Using both atomic and polyatomic projectiles for sputtering, such experiments have been carried out and some results have been briefly reported in our previous work [16]. In this work, we provide the overview and the interpretation of all results obtained. We present and discuss temperature dependencies of yields of sputtered homogeneous and heterogeneous cluster ions measured for a wide range of temperatures between 300 and $2400 \mathrm{~K}$ of the tantalum target bombarded by $12 \mathrm{keV}$ atomic $\mathrm{Au}^{-}$projectiles and by $18 \mathrm{keV}$ polyatomic $\mathrm{Au}_{3}{ }^{-}$projectiles.

\section{Experimental}

The secondary ion mass spectrometer (SIMS) used in the experiments is described in detail elsewhere $[10,11]$. To study the cluster ion emission under atomic and polyatomic ion bombardment, the commercial MI-1201 magnetic sector instrument (manufactured by SELMI, Sumy, Ukraine) was modified into the SIMS machine. To this end, it was equipped with a sputter ion source [17], primary ion column, target assembly with a heater, and ion optics for extracting secondary ions and delivering them into the mass spectrometer. The primary ion column included a mass separator and ion optics for focusing primary ion beams. Primary ions $(12 \mathrm{keV}$ $\mathrm{Au}^{-}$and $18 \mathrm{keV} \mathrm{Au}_{3}{ }^{-}$) bombarded the target surface at an incidence angle of $45^{\circ}$. Typical ion currents were $20 \mathrm{nA}$ for atomic projectiles, and $6 \mathrm{nA}$ for polyatomic ones. Polycrystalline tantalum was selected as the target because this was a mono-isotopic refractory material with high melting point $(3290 \mathrm{~K})$, which permitted the measurement of temperature dependencies of secondary ion yields within a wide temperature range. The target was prepared in a ribbon shape cut of a thin shim stock (length: $35 \mathrm{~mm}$, width: $3 \mathrm{~mm}$, and thickness: $30 \mu \mathrm{m}$ ). The chosen target ribbon aspect ratio (length $\gg$ width) assured homogeneity of a temperature distribution near the primary ion beam spots $(\approx 1 \mathrm{~mm}$ in diameter) located in the center of the target.

The residual gases pressure did not exceed $10^{-7}$ Torr under the experimental conditions. Since there was no residual gas analyzer available, simple estimates have been made to see what would be the possible oxygen coverage of sputtered surfaces if the assumed partial pressure of oxygen has been exaggerated to $100 \%$. For vacuum conditions indicated above, the surface arrival rate of oxygen atoms can be estimated by the known Hertz-Knudsen formula as $\sim 5 \times$ 
$10^{13} \mathrm{~cm}^{-2} \mathrm{~s}^{-1}$. For the primary ion current density of $\sim 2.5 \times$ $10^{-6} \mathrm{Acm}^{-2}$ and the sputtering yield of approximately five both corresponding to the atomic ion bombardment, the surface atom removal rate is estimated as $\sim 8 \times 10^{13} \mathrm{~cm}^{-2} \mathrm{~s}^{-1}$. Assuming sticking coefficient of 0.1 [18], one can then estimate the oxygen coverage at $T=300 \mathrm{~K}$ as $\left(0.15 \times 10^{13}\right) /((8$ $\left.\left.\times 10^{13}\right)+\left(0.15 \times 10^{13}\right)\right) \approx 7 \%$. This value should be considered as the very upper limit for atomic ion bombardment. For polyatomic ion bombardment, one can expect it to be scaled down by the increase of the sputtering yield. Moreover, increasing the target temperature can lower the sticking coefficient, which should decrease the oxygen coverage too.

During the measurements, this temperature was monitored using a pyrometer pointed at the primary ion beam spot on the target through one of the vacuum chamber viewports. The measured temperatures were corrected on the emissivity using tabulated data available from the literature [19]. Since oxygen coverage of surfaces was estimated to be low, no influence of surface oxygen on the emissivity was assumed, and therefore no corresponding correction has been applied. The accuracy of temperature measurements was about $\pm 40 \mathrm{~K}$.

To clean the target surface before the measurements, it was kept for several hours at a temperature of $T \approx 2500 \mathrm{~K}$ and simultaneously cleaned by the $12 \mathrm{keV} \mathrm{Au}^{-}$ion bombardment. For 20 different target temperatures $T$ in the range of $300 \mathrm{~K}$ $\leq T \leq 2400 \mathrm{~K}$, mass spectra of positive secondary ions have been measured in an analogue mode within the mass range of 0-2600 amu, keeping all other alignments of the SIMS instrument constant. The temperature dependencies of sputtered positive ion yields were extracted from these mass spectra. The errors of determining peak heights were about $5-10 \%$.

\section{Results and discussion}

The dependence of cluster ion yields on the target temperature $T$ was studied in two stages: (1) experiments at two temperatures aimed to obtain an overview, and (2) experiments to measure the temperature dependencies in detail.

\subsection{Overview experiments}

In the first stage, mass spectra of secondary ions sputtered from tantalum by $12 \mathrm{keV}$ atomic $\mathrm{Au}^{-}$and $18 \mathrm{keV}$ polyatomic $\mathrm{Au}_{3}{ }^{-}$projectiles were studied for two target temperatures, $T$ $=300$ and $2300 \mathrm{~K}$. Various peaks in the mass spectra were identified that corresponded to the target material (secondary $\mathrm{Ta}_{n}{ }^{+}$ions) as well as to the target impurities such as originally present niobium (secondary $\mathrm{Nb}^{+}$ions) and those introduced by the primary ion beam (secondary $\mathrm{Au}^{+}$ions). Moreover, heterogeneous cluster secondary ions such as $\mathrm{Ta}_{n} \mathrm{Nb}^{+}$and $\mathrm{Ta}_{n} \mathrm{O}_{m}{ }^{+}$were also identified in the mass spectra. As can see from Figs. 1-6, at $T=300$ and $2300 \mathrm{~K}$, these main types of sputtered ions demonstrated the following behaviour:

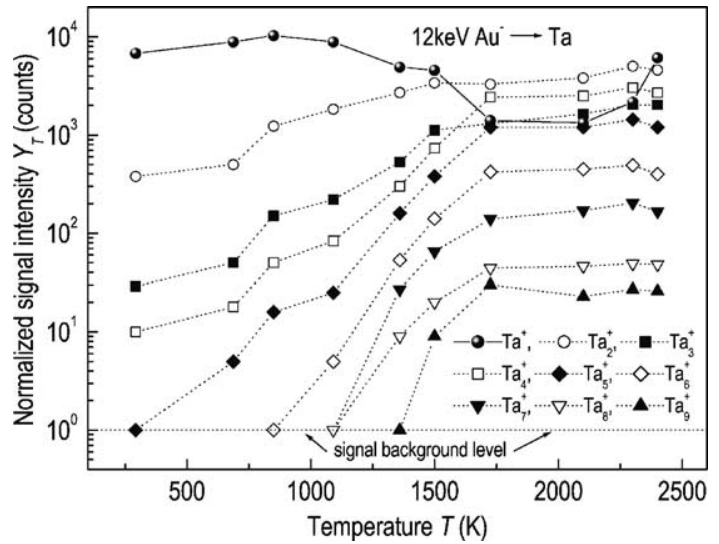

Fig. 1. Dependence of the $\mathrm{Ta}_{n}{ }^{+}$ion $(n=1-9)$ yields $Y_{T}$ on the target temperature $T$ for bombardment of tantalum with $12 \mathrm{keV}$ atomic $\mathrm{Au}^{-}$projectiles.

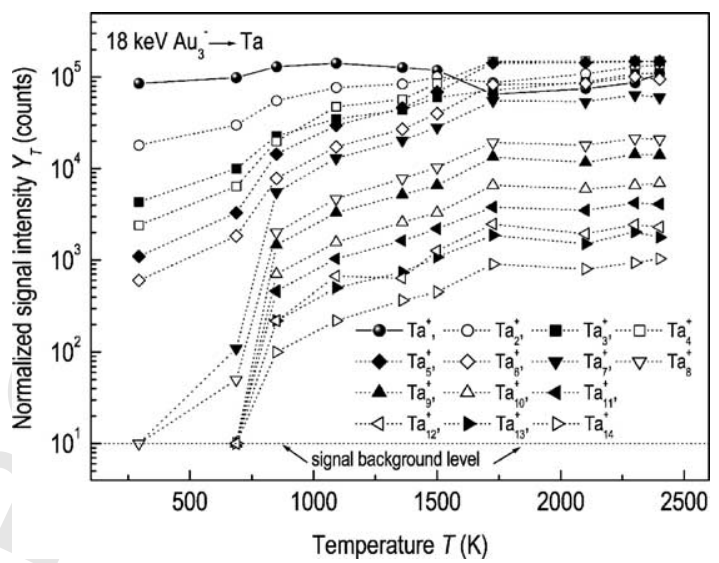

Fig. 2. Dependence of the $\mathrm{Ta}_{n}{ }^{+}$ion $(n=1-14)$ yields $Y_{T}$ on the target temperature $\mathrm{T}$ for bombardment of tantalum with $18 \mathrm{keV}$ polyatomic $\mathrm{Au}_{3}{ }^{-}$ projectiles.

(1) At $T=300 \mathrm{~K}$, mass spectra measured under atomic ion bombardment displayed peaks of the $\mathrm{Ta}_{n}{ }^{+}(n=1-4)$, $\mathrm{Nb}^{+}$and $\mathrm{Ta}_{n} \mathrm{O}_{m}{ }^{+}(m=1-3)$ ions. The same type of sputtered ions was observed under polyatomic ion bom-

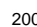

201 202 203

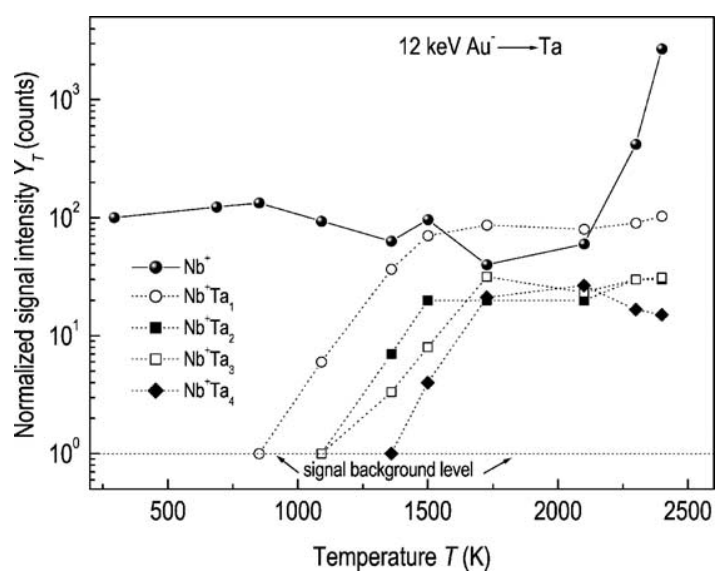

Fig. 3. Dependence of the $\mathrm{Nb}^{+}$and $\mathrm{Ta}_{n} \mathrm{Nb}^{+}$ion $(n=1-4)$ yields $Y_{T}$ on the target temperature $T$ for bombardment of tantalum with $12 \mathrm{keV}$ atomic $\mathrm{Au}^{-}$ projectiles. 


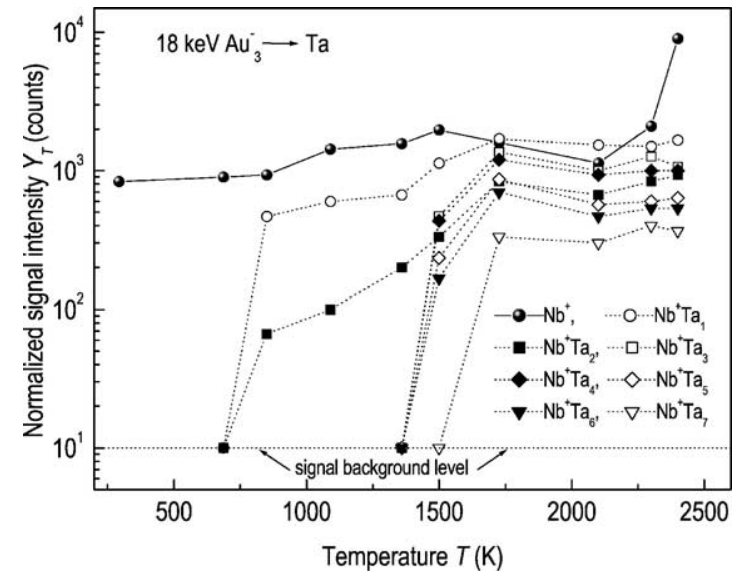

Fig. 4. Dependence of the $\mathrm{Nb}^{+}$and $\mathrm{Ta}_{n} \mathrm{Nb}^{+}$ion $(n=1-7)$ yields $Y_{T}$ on the target temperature $T$ for bombardment of tantalum with $18 \mathrm{keV}$ polyatomic $\mathrm{Au}_{3}{ }^{-}$projectiles.

bardment. The difference between spectra acquired for atomic and polyatomic projectiles was that the higher intensities of sputtered ions produced by the $\mathrm{Au}_{3}{ }^{-}$bombardment permitted the detection of $\mathrm{Ta}_{5}{ }^{+}$and $\mathrm{Ta}_{6}{ }^{+}$ions. For both projectiles, the $\mathrm{Ta}_{n}{ }^{+}$ion intensities decreased monotonously with increasing $n$.

(2) At $T=2300 \mathrm{~K}$, all peaks of $\mathrm{Ta}_{n} \mathrm{O}_{m}{ }^{+}$ions have disappeared from the mass spectra. For atomic ion bombardment, $\mathrm{Ta}_{n}{ }^{+}(n=1-10), \mathrm{Nb}^{+}$and $\mathrm{Ta}_{n} \mathrm{Nb}^{+}(n=1-4)$ ions were detected. The same ions were observed in the mass spectra under polyatomic bombardment: $\mathrm{Ta}_{n}{ }^{+}(n$ $=1-14), \mathrm{Nb}^{+}$and $\mathrm{Ta}_{n} \mathrm{Nb}^{+}(n=1-8)$ ions. For atomic bombardment, the $\mathrm{Ta}_{2}{ }^{+}$ions showed the highest intensities, while, for polyatomic bombardment, the $\mathrm{Ta}_{4}{ }^{+}$ions were the most intense. In agreement with our previous results [12,13], $\mathrm{Ta}_{n}{ }^{+}$ion intensities at $T=2300 \mathrm{~K}$ decreased with increasing $n$ starting from the above most intense cluster ions ( $n=2$ and 4$)$.

(3) At $T=2300 \mathrm{~K}$, the $\mathrm{Ta}_{n}{ }^{+}$ion signals were higher than those measured at $T=300 \mathrm{~K}$. They increased by factors of

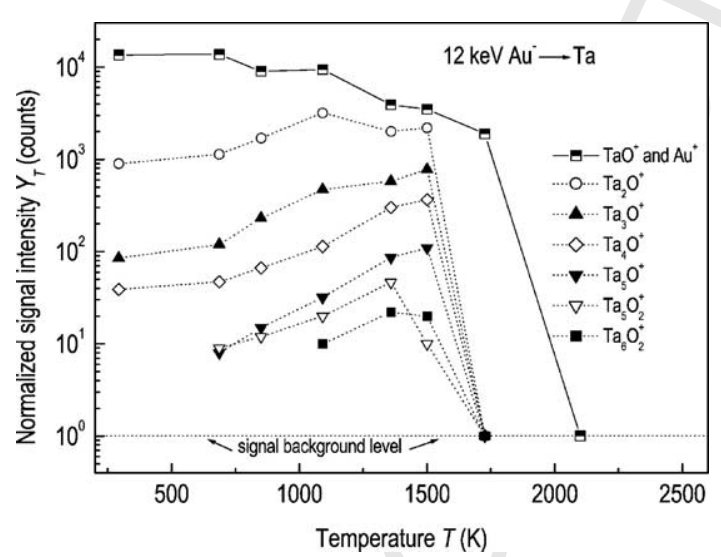

Fig. 5. Dependencies of the $\mathrm{Au}^{+}$and $\mathrm{TaO}^{+}, \mathrm{Ta}_{n} \mathrm{O}^{+}(n=2-5)$, and $\mathrm{Ta}_{n} \mathrm{O}_{2}{ }^{+}$ $(n=5,6)$ ion yields $Y_{T}$ on the target temperature $T$ for bombardment of tantalum with $12 \mathrm{keV}$ atomic $\mathrm{Au}^{-}$projectiles.
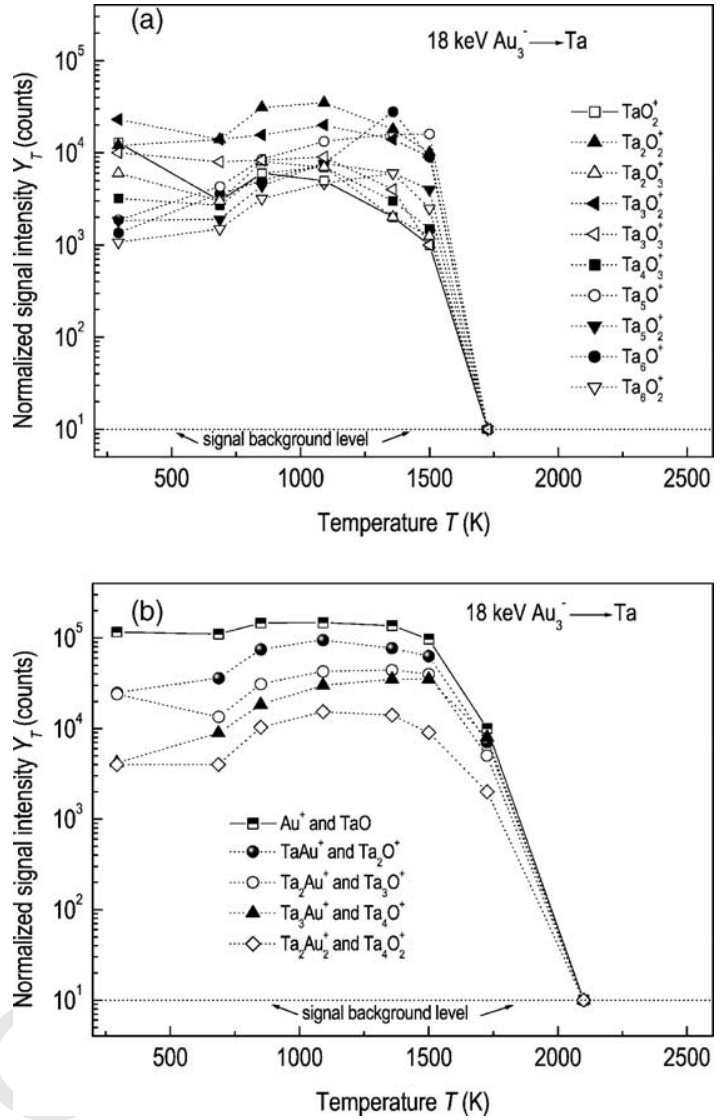

Fig. 6. (a) Dependence of the $\mathrm{Ta}_{n} \mathrm{O}_{m}{ }^{+}(n=1-6 ; m=1-3)$ ion yields $Y_{T}$ on the target temperature $T$ for bombardment of tantalum with $18 \mathrm{keV}$ polyatomic $\mathrm{Au}_{3}{ }^{-}$projectiles. (b) Dependencies of the $\mathrm{Au}^{+}$and $\mathrm{TaO}^{+}, \mathrm{TaAu}^{+}$ and $\mathrm{Ta}_{2} \mathrm{O}^{+}, \mathrm{Ta}_{2} \mathrm{Au}^{+}$and $\mathrm{Ta}_{3} \mathrm{O}^{+}, \mathrm{Ta}_{3} \mathrm{Au}^{+}$and $\mathrm{Ta}_{4} \mathrm{O}^{+}$as well as $\mathrm{Ta}_{2} \mathrm{Au}_{2}{ }^{+}$ and $\mathrm{Ta}_{4} \mathrm{O}_{2}{ }^{+}$ion yields $Y_{T}$ on the target temperature $T$ for bombardment of tantalum with $18 \mathrm{keV}$ polyatomic $\mathrm{Au}_{3}{ }^{-}$projectiles.

10 for $\mathrm{Ta}_{2}{ }^{+}, 35$ for $\mathrm{Ta}_{3}{ }^{+}$, and 200 for $\mathrm{Ta}_{4}{ }^{+}$for sputtering with the $\mathrm{Au}^{-}$projectiles and by factors of 5 for $\mathrm{Ta}_{2}{ }^{+}$, 25 for $\mathrm{Ta}_{3}{ }^{+}, 50$ for $\mathrm{Ta}_{4}{ }^{+}, 100$ for $\mathrm{Ta}_{5}{ }^{+}$, and $\mathrm{Ta}_{6}{ }^{+}$for sputtering with the $\mathrm{Au}_{3}{ }^{-}$projectiles.

Thus, the mass spectra of secondary ions clearly depended on both the target temperature and the projectile type. Changing these two variables led to the redistribution of peak patterns and intensities in the mass spectra. The origin of these changes is presently unclear and debatable, and one can only hope that future studies will help to fully understand it. Easily recognizable from these data was a clearly different behaviour in signals of the $\mathrm{Ta}_{n}{ }^{+}$and $\mathrm{Ta}_{n} \mathrm{O}_{m}{ }^{+}$ions while increasing the target temperature $T$. For example, the emission of cluster ions $\mathrm{Ta}_{n}{ }^{+}$with $n>4$ for atomic projectiles and that with $n>6$ for polyatomic projectiles were observed at $T=2300 \mathrm{~K}$, but not detected at $T=300 \mathrm{~K}$. In contrast, intense signals of $\mathrm{Ta}_{n} \mathrm{O}_{m}{ }^{+}$ions at $T=300 \mathrm{~K}$ completely disappeared at $T=2300 \mathrm{~K}$. This suggests that the $\mathrm{Ta}_{n} \mathrm{O}_{m}{ }^{+}$ ion emission might be one of the limiting factors influencing the yield of $\mathrm{Ta}_{n}{ }^{+}$cluster ions at low (room) temperature.
224 225 226 227 228 229 230 231 232 233 234 235 236 237 238 239 240 241 242 243 


\subsection{Temperature dependence}

The effort to better understand this process stimulated the second stage of experiments. For $12 \mathrm{keV} \mathrm{Au}^{-}$and $18 \mathrm{keV}$ $\mathrm{Au}_{3}{ }^{-}$ion bombardments, the mass spectra were measured in the same manner, as described above, for various target temperatures within the range of $300 \mathrm{~K} \leq T \leq 2400 \mathrm{~K}$ so that dependencies of ion yields $Y_{T}$ versus $T$ could be produced from the experimental data. This was done for the following groups of ions: $\mathrm{Ta}_{n}{ }^{+}, \mathrm{Ta}_{n} \mathrm{Nb}^{+}, \mathrm{Ta}_{n} \mathrm{O}_{m}{ }^{+}$, and $\mathrm{Ta}_{n} \mathrm{Au}^{+}$. The measurement process took several days, so that the instrument was optimized for one sort of the primary ions. To compare signal intensities measured during different experimental sessions, the intensities obtained for the same projectile were normalized to the primary ion current. Thus in figures shown below signal intensities under bombardment with the same projectile can be compared with a reasonable accuracy. As for the comparison between data acquired with different primary ions that had, in addition, different kinetic energies, here we were not particularly interested to quantitatively compare emissions under $12 \mathrm{keV} \mathrm{Au}^{-}$and $18 \mathrm{keV}$ $\mathrm{Au}_{3}{ }^{-}$projectiles since we reported on that in Ref. [10]. Due to the longevity of the measurement process mentioned above and the differences in dynamics of sputtering and surface cleaning processes for atomic and polyatomic ion bombardment we preferred not to compare these cases directly.

\subsubsection{The temperature dependence of $\mathrm{Ta}_{n}{ }^{+}$ion yield}

The temperature dependencies of the $\mathrm{Ta}_{n}{ }^{+}$yields $Y_{T}$ obtained under atomic and polyatomic ion bombardments are shown in Figs. 1 and 2, respectively. One can recognize the following trends:

(1) For atomic ions $\mathrm{Ta}^{+}$, temperature dependencies demonstrate a similar behaviour for either projectiles, as follows: $Y_{T}$ slightly increases with increasing temperature up to $T \approx 900 \mathrm{~K}$, then $Y_{T}$ starts decreasing and reaches a plateau at $T \approx 1700 \mathrm{~K}$, until finally $Y_{T}$ increases again at $T$ $>2300 \mathrm{~K}$. In a SIMS instrument with (ultra) high vacuum conditions, the yield of the $\mathrm{Ta}^{+}$ions can be dependent on the target temperature because an elevated $T$ generally helps to clean the target surface from chemicallyreactive impurities such as oxygen or alkali metals that can strongly influence the surface electronic properties. According to a well-established and accepted theoretical interpretation described by $\mathrm{Yu}$ [2], charge state formation occurs due to the electron exchange process between the departing sputtered atom and the surface. This process depends on the elemental species involved, the velocity and the angle of motion of the ejected atom, and the electronic properties of the surface. The oxygen concentration of the sputtered surface depends on the competition between processes bringing oxygen onto the surface (such as the oxygen adsorption from residual gases and thermal diffusion of the dissolved oxygen from the bulk), and those removing oxygen from the surface (such as thermal desorption and ion sputtering). Moreover, the sputtering yield alone varies with the thickness of the oxide layer on the metal surface because metal oxides typically have lower sputtering yield than metals themselves. In view of this, one can explain the temperature dependence of the $\mathrm{Ta}^{+}$ion emission shown in Figs. 1 and 2 , as follows. Under our experimental conditions, oxygen is apparently present on the sputtered tantalum surface at temperatures $T<1700 \mathrm{~K}$ so that for the $\mathrm{Ta}^{+}$ions $Y_{T}$ displays the temperature dependence observed. Cleaning the sample surface by ion bombardment together with heating could somewhat increases the sputtering yield of Ta because of a more efficient removal of the oxide film. On the other hand, the decreased concentration of oxygen on the Ta surface lowers the ionization probability. The competition between these two factors that both affect the detected $\mathrm{Ta}^{+}$ion signals might produce the variation observed: a slight rise with a maximum at temperatures $\sim 1000 \mathrm{~K}$ and then a decrease. For atomic ion bombardment, $Y_{T}$ decreases overall by a factor of five over a temperature interval from $T=300$ to $1700 \mathrm{~K}$. A smaller $\mathrm{Ta}^{+}$yield decrease by a factor of two is observed under polyatomic ion bombardment. This suggests that the nonlinear increase in the sputtering yield under polyatomic ion bombardment (the non-additive effect) has a stronger influence on the detected signals than the decrease in the ionization probability of sputtered atoms due to the removal of oxygen. Moreover, one could expect that since polyatomic projectiles produce higher sputtering yields, they clean the surface more efficiently. In general, under both atomic and polyatomic bombardments, it can be observed that the coverage of the tantalum surface by oxygen atoms is not very dense because its change as a result of the temperature increase from $T=300$ to $1700 \mathrm{~K}$ does not affect the $\mathrm{Ta}^{+}$yields dramatically. In this context, a plateau of the $Y_{T}$ curves in the range of $1700 \mathrm{~K}$ $<T<2300 \mathrm{~K}$ likely corresponds to $\mathrm{Ta}^{+}$ions sputtered from the clean free surface.

For $T>2300 \mathrm{~K}$, an increase of $Y_{T}$ is observed due to the contribution of the thermal ionization (when evaporated atoms are ionized on the hot metal surface). In our present and previous experiments [10-12], substantial currents of such evaporated $\mathrm{Ta}^{+}$ions were observed at $T>2300 \mathrm{~K}$ in absence of ion bombardment. At $T=$ $2400 \mathrm{~K}$, these signals were almost equal to those of sputtered $\mathrm{Ta}^{+}$ions. Kinetic energy distributions of the evaporated $\mathrm{Ta}^{+}$ions are symmetric and narrow compared with those for the sputtered $\mathrm{Ta}^{+}$ions. Typically, full widths at half maximum (FWHM) for the distribution of evaporated and sputtered $\mathrm{Ta}^{+}$ions are about $7.2 \mathrm{eV}$ and a few tens $\mathrm{eV}$, respectively [11]. This rather large value of FWHM for the experimental energy distribution of evaporated $\mathrm{Ta}^{+}$ions is determined by an instrumental effect. It results from the convolution of an original energy distribution of evaporated ions (FWHM is of the order of $k T \approx 0.2 \mathrm{eV} ; k$ is Boltzmann constant) with the appara- 
tus response function. This allowed us to determine the energy resolution of $\sim 7 \mathrm{eV}$ (at FWHM) for our SIMS instrument operated in measurement conditions with partly open slits for increased sensitivity.

Thus, in the range of $300 \mathrm{~K}<T<2300 \mathrm{~K}$, the temperature dependence of the $\mathrm{Ta}^{+}$ion yield stays in good agreement with the typical dependence of sputtered ions yields on the degree of oxygen coverage of metal surfaces. It can be used, as a reference curve, when temperature dependence of cluster ion yields come in the focus of our discussion.

(2) For $\mathrm{Ta}_{n}{ }^{+}$ions, the variation of $Y_{T}$ in the range of $T<$ $1700 \mathrm{~K}$ depends on the projectile species. Under atomic ion bombardment, the increase of $T$ in the range of $300 \mathrm{~K}$ $<T<1700 \mathrm{~K}$ strongly enhances the signal intensities of $\mathrm{Ta}_{n}{ }^{+}$cluster ions $(2<n<10)$ so that at certain temperatures additional peaks appear in the mass spectrum corresponding to larger $\mathrm{Ta}_{n}{ }^{+}$cluster ions with $(n>4)$. The temperature increase also causes changes in the cluster ion yield distribution. For example, the ratio $Q=$ $Y_{T}\left(\mathrm{Ta}_{2}{ }^{+}\right) / Y_{T}\left(\mathrm{Ta}_{4}{ }^{+}\right)$is $\approx 40$ at $T=300 \mathrm{~K}$ and changes to $Q \approx 1.5$ for $T>1700 \mathrm{~K}$. Under polyatomic ion bombardment, this effect is even more pronounced: the $Q$ value changes from $Q \approx 8$ at $T=300 \mathrm{~K}$ to $Q \approx 0.7$ at $T$ $>1700 \mathrm{~K}$ thus showing that the maximum of the cluster yield distribution is shifting with temperature to larger clusters. Under polyatomic ion bombardment, peaks of $\mathrm{Ta}_{n}{ }^{+}$cluster ions with $n>6$ appear in the spectra at lower temperatures than for atomic bombardment. For instance, $\mathrm{Ta}_{7}{ }^{+}$appears at $T \approx 700 \mathrm{~K}$ compared to $T \approx$ $1350 \mathrm{~K}$ for atomic projectiles. This can be considered as an evidence of more efficient formation/emission of larger homogeneous clusters $\mathrm{Ta}_{n}{ }^{+}$under polyatomic ion bombardment.

In Fig. 1 it appears that slopes of the $Y_{T}$ curves become steeper with increasing cluster size, thus demonstrating a stronger influence of the surface cleanliness on the emission of larger cluster ions. On the other hand, in Fig. 2 these slopes are similar thus supporting the hypothesis that polyatomic projectiles are more efficient in cleaning the surface.

(3) In contrast to atomic $\mathrm{Ta}^{+}$ions, no evaporated cluster $\mathrm{Ta}_{n}{ }^{+}$ions $(n=2-14)$ were observed at $T>2300 \mathrm{~K}$. This shows how significant the differences are between the energy deposition and dissipation in ion sputtering and thermal evaporation processes. Compared with the nonthermal sputtering mechanism, a thermal excitation of tantalum alone could not create conditions to initiate and stimulate cluster ion formation and emission processes.

(4) For both atomic and polyatomic projectiles, the signals of the $\mathrm{Ta}_{n}{ }^{+}$secondary ions display their dependence on the target temperature only for $T<1700 \mathrm{~K}$. For the temperatures $T>1700 \mathrm{~K}$, i.e. when there is no oxygen on the tantalum surface, signals of all identified $\mathrm{Ta}_{n}{ }^{+}$ions reach their saturation, which manifests itself as an appearance of plateaus on the corresponding $Y_{T}$ curves. As- suming this ionization probability to be high for sputtering of clean Ta surfaces [9] and seeing no apparent signal changes in $Y_{T}$, one can conclude that both the sputtering yield of neutral clusters and the probability of their ionization do not depend on temperature in range of $1700 \mathrm{~K}$ $\leq T \leq 2400 \mathrm{~K}$. This generates a question: what processes could cause the temperature dependence of the $\mathrm{Ta}_{n}{ }^{+}$ion signals for temperatures lower than $1700 \mathrm{~K}$ ? The temperature dependence measured for heterogeneous (mixed) cluster ions such as those containing niobium, oxygen and gold atoms as additions to tantalum might help to shed more light on this.

\subsubsection{The temperature dependence of $\mathrm{Ta}_{n} \mathrm{Nb}^{+}$ion yield}

Temperature dependence of $Y_{T}$ for mixed cluster ions containing niobium $\mathrm{Ta}_{n} \mathrm{Nb}^{+}$measured under atomic and polyatomic ion bombardment are shown in Figs. 3 and 4, respectively. Ion emission from tantalum in the form of the $\mathrm{Nb}^{+}$ and $\mathrm{Ta}_{n} \mathrm{Nb}^{+}$ions occurs due to a presence of a low concentration of niobium impurity $(<0.01 \%)$ in the Ta sample. At $T$ $=300 \mathrm{~K}$, no peaks of the $\mathrm{Ta}_{n} \mathrm{Nb}^{+}$ions are observed, and only the $\mathrm{Nb}^{+}$ion peak is detectable. The signals of $\mathrm{Nb}^{+}$ions behave similarly to those for the $\mathrm{Ta}^{+}$ions (Figs. 1 and 2): $\mathrm{Nb}^{+}$ peak intensities slightly rise and then fall with temperature in the range of $300 \mathrm{~K} \leq T \leq 1700 \mathrm{~K}$. At the same time, peaks of the $\mathrm{Ta}_{n} \mathrm{Nb}^{+}$ions appear in the mass spectra. Such clusters with $n \leq 4$ and 8 , are identified for atomic and polyatomic ion bombardments, respectively. In the range of $300 \mathrm{~K} \leq T$ $\leq 1700 \mathrm{~K}$, the signal intensities increase with temperature and then reach saturation at $T>1700 \mathrm{~K}$ for both atomic and polyatomic bombardment. In contrast to these mixed $\mathrm{Ta}_{n} \mathrm{Nb}^{+}$ clusters, signals of atomic $\mathrm{Nb}^{+}$ions show a strong increase at temperatures $T>2300 \mathrm{~K}$, which can be explained in the same way as for $\mathrm{Ta}^{+}$ions, by the emission of evaporated ions in addition to the ion sputtering. In general, the temperature dependence obtained for $\mathrm{Nb}^{+}$and $\mathrm{Ta}_{n} \mathrm{Nb}^{+}$ions are similar to those obtained for $\mathrm{Ta}_{n}{ }^{+}$ions. This means that the substitution of one tantalum atom in $\mathrm{Ta}_{n}{ }^{+}$clusters by one niobium atom (with similar physical-chemical properties) does not dramatically change neither cluster properties nor emission and charge state formation mechanisms. Essentially, these mixed heterogeneous $\mathrm{Ta}_{n-1} \mathrm{Nb}^{+}$clusters behave in the same way as do homogeneous $\mathrm{Ta}_{n}{ }^{+}$clusters. It should be noted that the existence of the $\mathrm{Ta}_{n} \mathrm{Nb}^{+}$emission channel decreases the weight of the $\mathrm{Ta}_{n}{ }^{+}$channel thus attenuating the signals detected. It is yet unclear whether this attenuation is temperature dependent or not.

\subsubsection{The temperature dependence of $\mathrm{Ta}_{n} \mathrm{O}_{m}{ }^{+}$and $\mathrm{Ta}_{n} \mathrm{Au} u^{+}$yield}

The temperature dependencies of the $\mathrm{Ta}_{n} \mathrm{O}_{m}{ }^{+}$and $\mathrm{Ta}_{n} \mathrm{Au}^{+}$ion yields $Y_{T}$ measured under atomic and polyatomic ion bombardment are shown in Figs. 5 and $6 a$ and b, respectively. From a variety of such secondary ions observed in our experiments, we chose the ones with peak intensities sufficient to measure their emission within a wide range of target 
temperatures. An identification of these peaks is not easy because the bombardment of tantalum with gold projectiles can both implant and then sputter the implanted gold atoms, which results in the emission of the $\mathrm{Au}^{+}$and $\mathrm{Ta}_{n} \mathrm{Au}_{m}{ }^{+}$ions. For these ions, the mass-to-charge ratios $m / q$ are very close to those of $\mathrm{Ta}_{n} \mathrm{O}_{m}{ }^{+}$ions. For example, the $m / z=197$ peak can be attributed to both $\mathrm{TaO}^{+}(m / q=181+16=197)$ and $\mathrm{Au}^{+}$ $(m / q=197)$ ions. An isobaric interference between peaks of ${ }^{181} \mathrm{Ta}_{n-1}{ }^{197} \mathrm{Au}^{+}$and ${ }^{181} \mathrm{Ta}_{n}{ }^{16} \mathrm{O}^{+}$ions can occur because $\mathrm{m} / \mathrm{q}$ $=180.9479(n-1)+196.96655 \approx 180.9479 n+15.99491$, and in order to resolve them, one would need a SIMS instrument with mass resolution exceeding 8500 . This is impossible for our instrument operated in the regime with partly open slits to detect weak signals [12]. In such a measurement mode, it was very difficult to use other peak identification methods, for example, observing ${ }^{181} \mathrm{Ta}_{n}{ }^{18} \mathrm{O}_{m}{ }^{+}$ions in parallel with ${ }^{181} \mathrm{Ta}_{n}{ }^{16} \mathrm{O}_{m}{ }^{+}$ions in order to separate the $\mathrm{Ta}_{n} \mathrm{O}_{m}{ }^{+}$ion contribution from that of $\mathrm{Ta}_{n} \mathrm{Au}_{m}{ }^{+}$. Unfortunately, due to the low natural abundance of ${ }^{18} \mathrm{O}$ isotopes $(\approx 0.2 \%)$ and insufficiently wide dynamic range of our SIMS instrument, it was impossible to measure the $Y_{T}$ dependencies for ${ }^{181} \mathrm{Ta}_{n}{ }^{18} \mathrm{O}_{m}{ }^{+}$ ions within sufficiently wide range, especially near the most interesting temperatures of $T \approx 1700 \mathrm{~K}$. As can be seen below, at this temperature it might be possible to distinguish between $\mathrm{Ta}_{n} \mathrm{O}_{m}{ }^{+}$and $\mathrm{Ta}_{n} \mathrm{Au}_{m}{ }^{+}$secondary ions.

Under the $\mathrm{Au}^{-}$atomic ion bombardment (Fig. 5), temperature dependencies for yields of secondary ions with $m / q=$ 378, 559, 740, 921, 937, and 1118 reveal similar behaviour. Their intensities increase with the temperature, reach their maximums at $T \approx 1500 \mathrm{~K}$, and then begin to decrease falling to zero at $T \approx 1700 \mathrm{~K}$. Such behaviour of the temperature dependencies we will call "normal". It is important to realize that the zeroing signals of the above ions occurs at the same temperature $(T \approx 1700 \mathrm{~K})$ where the plateau appears on temperature dependencies $Y_{T}$ of $\mathrm{Ta}_{n}{ }^{+}$and $\mathrm{Ta}_{n} \mathrm{Nb}^{+}$ions (Figs. 1-4). This coincidence of the disappearance of one sort of ions with the "stabilization" of the yield of the other ions might indicate a redistribution of material between different emission channels. At the same time, for ions with $m / q=197$, the $Y_{T}$ dependence exhibits an "abnormal" behaviour when the ion signal intensity slightly increases with temperature in the range of $300 \mathrm{~K}<T<1700 \mathrm{~K}$ and for $T>1700 \mathrm{~K}$ starts to sharply decrease reaching zero at $T \approx 2100 \mathrm{~K}$.

Compared with the atomic ion bombardment, the $\mathrm{Au}_{3}{ }^{-}$ cluster ion bombardment (Fig. 6a and b) generates a wider variety of sputtered cluster ions. Among them, one can recognize ions with both "normal" and "abnormal" temperature dependencies $Y_{T}$. One group of ions with $m / q=213,394,410$, $575,591,772,921,937,1102$, and 1118 , shows the "normal" dependencies $Y_{T}$. Intensities of these ions decrease to zero at $T \approx 1700 \mathrm{~K}$ (Fig. 6a). The other group behaves "abnormally": the intensities of ions with $m / q=197,378,559,740$, and 756 decrease to zero at $T \approx 2100 \mathrm{~K}$ (Fig. 6b).

The comparison of the $Y_{T}$ curves measured under atomic and polyatomic ion bombardments shows that: (1) for ions with $m / q=197$, only "abnormal" temperature dependencies are observed in either case. (2) On the contrary, for secondary ions with $m / q=378,559$, and 740 , the behaviour of $Y_{T}$ dependencies is changed from "normal" to "abnormal" when the atomic projectiles are replaced by the polyatomic ones. (3) Secondary ions with $m / q=756$ are observed only under polyatomic ion bombardment and exhibit the "abnormal" behaviour of the $Y_{T}$ dependence.

According to mass spectrometric data reported in Ref. [20], while a tantalum ribbon is heated to high temperatures, the oxygen removal from the metal surface occurs mainly in form of the thermal desorption of $\mathrm{TaO}$ molecules. Using Auger electron spectroscopy, it was shown in Ref. [21] that the tantalum ribbon not exposed to gaseous environments (such as plasma) did not contain bulk contaminants, and the surface impurities (such as oxygen, carbon and sulphur) were completely eliminated at $T>1300 \mathrm{~K}$. However, if the ribbon contained oxygen in its bulk then its removal from Ta occurs at much higher temperatures. For example, the complete removal of an oxide monolayer (that corresponds to the surface oxygen concentration of $7 \times 10^{14} \mathrm{~cm}^{-2}$ ) from Ta at $T=$ $2350 \mathrm{~K}$ takes about $60 \mathrm{~s}$ [22]. In our experiments, heated tantalum surfaces were exposed to ion bombardments that were cleaning the surfaces, on one hand, but could also cause ion implantation and ion mixing, on the other. The ion mixing phenomena could redistribute some surface oxygen atoms into the sub-surface regions of the target, and the ion implantation could also distribute gold atoms in the same regions. Compared with the $12 \mathrm{keV} \mathrm{Au}^{-}$bombardment, the $18 \mathrm{keV}$ $\mathrm{Au}_{3}{ }^{-}$bombardment produces much higher concentrations of gold implants due to their shorter ion ranges (because of a lower energy per atom) and tripled numbers of atoms per projectile. Taking all these phenomena into consideration, we came up with the following interpretation of our results.

We hypothesize that the characteristic temperature of $T \approx 1700 \mathrm{~K}$ corresponds to a complete removal of oxide molecules from the target. In this case, in Fig. 5 secondary ions with $m / q=378,559,740,921,937$, and 1118 can be identified as $\mathrm{Ta}_{2} \mathrm{O}^{+}, \mathrm{Ta}_{3} \mathrm{O}^{+}, \mathrm{Ta}_{4} \mathrm{O}^{+}, \mathrm{Ta}_{5} \mathrm{O}^{+}, \mathrm{Ta}_{5} \mathrm{O}_{2}{ }^{+}$, and $\mathrm{Ta}_{6} \mathrm{O}_{2}{ }^{+}$, respectively. In the same way, in Fig. 6, secondary ions with $m / q=213,394,410,575,591,772,921,937$, 1102 , and 1118 can be identified as $\mathrm{TaO}_{2}{ }^{+}, \mathrm{Ta}_{2} \mathrm{O}_{2}{ }^{+}, \mathrm{Ta}_{2} \mathrm{O}_{3}{ }^{+}$, $\mathrm{Ta}_{3} \mathrm{O}_{2}{ }^{+}, \mathrm{Ta}_{3} \mathrm{O}_{3}{ }^{+}, \mathrm{Ta}_{4} \mathrm{O}_{3}{ }^{+}, \mathrm{Ta}_{5} \mathrm{O}^{+}, \mathrm{Ta}_{5} \mathrm{O}_{2}{ }^{+}, \mathrm{Ta}_{6} \mathrm{O}^{+}$, and $\mathrm{Ta}_{6} \mathrm{O}_{2}{ }^{+}$, respectively.

On the other hand, one can expect higher desorption temperatures for gold atoms than for oxide molecules. Therefore at temperatures $T>1700 \mathrm{~K}$ the $m / q=197$ peaks exhibiting the "abnormal" behaviour of the $Y_{T}$ dependencies under atomic and polyatomic ion bombardments (see Figs. 5 and 6b), can be identified as predominantly $\mathrm{Au}^{+}$ions because a possible contribution of $\mathrm{TaO}^{+}$ions should then become insignificant. However, keeping in mind the significance of oxygen containing molecular secondary ions at $T<1700 \mathrm{~K}$ and assuming that the formation of $\mathrm{TaO}^{+}$ions is more probable in sputtering than that of other $\mathrm{Ta}_{n} \mathrm{O}_{m}{ }^{+}$ions, one should not neglect the $\mathrm{TaO}^{+}$ions in this temperature range. This leaves us with 
the conclusion that for $T<1700 \mathrm{~K}$ the $m / q=197$ peaks are formed by a sum of $\mathrm{Au}^{+}$and $\mathrm{TaO}^{+}$ion currents.

At the same time, the polyatomic ion bombardment makes a noticeable difference for secondary ions with $m / q=378$, 559 , and 740 by an apparent addition of new emission channels, such as $\mathrm{TaAu}^{+}, \mathrm{Ta}_{2} \mathrm{Au}^{+}$, and $\mathrm{Ta}_{3} \mathrm{Au}^{+}$that dominate at temperatures $T>1700 \mathrm{~K}$ when $\mathrm{Ta}_{n} \mathrm{O}_{m}{ }^{+}$ions disappear from the spectra (Fig. 6b). The possibility of initiating new emission channels by the polyatomic ion bombardment can be clearly seen from the appearance in the spectra of secondary ions with $m / q=756$ that exhibit the "abnormal" $Y_{T}$ dependence. While these peaks can be possibly created by both $\mathrm{Ta}_{2} \mathrm{Au}_{2}{ }^{+}$and $\mathrm{Ta}_{4} \mathrm{O}_{2}{ }^{+}$ions, at temperatures $T>1700 \mathrm{~K}$ (Fig. $6 b)$ one can expect them to be formed by mostly $\mathrm{Ta}_{2} \mathrm{Au}_{2}{ }^{+}$ ions because the $\mathrm{Ta}_{4} \mathrm{O}_{2}{ }^{+}$channel should not then have any significance.

Thus various emission channels observed under gold ion bombardment of tantalum $\left(\mathrm{Ta}_{n}{ }^{+}, \mathrm{Ta}_{n} \mathrm{Nb}^{+}, \mathrm{Ta}_{n} \mathrm{O}_{m}{ }^{+}\right.$, and $\mathrm{Ta}_{n} \mathrm{Au}^{+}$) have distinctive dependencies of their intensity on the target temperature. Moreover, one might notice that the emissions of the $\mathrm{Ta}_{n}{ }^{+} / \mathrm{Ta}_{n} \mathrm{Nb}^{+}$and $\mathrm{Ta}_{n} \mathrm{O}_{m}{ }^{+} / \mathrm{Ta}_{n} \mathrm{Au}^{+}$cluster ions are interrelated and depend on the surface concentrations of both oxide molecules and gold atoms. However, these are opposite trends: removing the oxide and gold atoms enhances the $\mathrm{Ta}_{n}{ }^{+}$and $\mathrm{Ta}_{n} \mathrm{Nb}^{+}$ion emission and suppresses the $\mathrm{Ta}_{n} \mathrm{O}_{m}{ }^{+}$and $\mathrm{Ta}_{n} \mathrm{Au}^{+}$emission, and vice versa, letting the oxide film and the gold atom coverage to grow suppresses $\mathrm{Ta}_{n}{ }^{+}$ and $\mathrm{Ta}_{n} \mathrm{Nb}^{+}$ion emissions and stimulates those of $\mathrm{Ta}_{n} \mathrm{O}_{m}{ }^{+}$ and $\mathrm{Ta}_{n} \mathrm{Au}^{+}$. It seems that these processes sense the actual surface composition (or the degree of surface cleanliness). It is important that tantalum atoms in the sputtered flux are redistributed between these various emission channels, and such redistribution depends on both the target temperature and the nature of primary ions (atomic or polyatomic).

\section{Summary and conclusion}

Results presented in Figs. 1-6 demonstrate the complexity of the spectrum of major emission components produced in sputtering of tantalum targets by atomic $\mathrm{Au}^{-}$and polyatomic $\mathrm{Au}_{3}{ }^{-}$ions, and how these emissions depend on target temperature. Conducting experiments under moderately high (but not ultra high) vacuum conditions of $10^{-7}$ Torr and varying both the target temperature over a range of $300 \mathrm{~K} \leq T \leq$ $2400 \mathrm{~K}$ and the projectile type permits studies of the influence of surface conditions on the emission of positive secondary ions. In addition to the atomic secondary ions $\mathrm{Ta}^{+}$, $\mathrm{Nb}^{+}$and $\mathrm{Au}^{+}$, two types of secondary cluster ions were observed, namely homogenous clusters $\mathrm{Ta}_{n}{ }^{+}$, and various heterogeneous (mixed) clusters such as $\mathrm{Ta}_{n} \mathrm{Nb}^{+}, \mathrm{Ta}_{n} \mathrm{Au}^{+}$, and $\mathrm{Ta}_{n} \mathrm{O}_{m}{ }^{+}$. It seems reasonable to assume that the mixed cluster ion formations result from competing reactions including an association of the Ta atoms and the atoms of impurities or bulk/surface contaminants such as the $\mathrm{Nb}, \mathrm{Au}$, and $\mathrm{O}$ atoms. The efficiency of these reactions depends on the activation energies that reflect the relative reactivity of interacting atoms, the equilibrium concentration of impurity atoms in the subsurface region of the emission spot, which depends on the type and current of projectiles, and the size and geometry of complex ions. One can also expect that both the reaction activation energies and the surface concentrations of impurity/contamination atoms depend on the target temperature.

In comparison to niobium and gold, oxygen atoms demonstrate higher reactivity for $\mathrm{Ta}$ atoms, and the oxygencontaining $\mathrm{Ta}_{n} \mathrm{O}_{m}{ }^{+}$ion emission channel is substantial at room temperatures. A similar effect has been observed in an excited molecule emission from tantalum produced by the $3 \mathrm{keV} \mathrm{Ho}{ }^{+}$bombardment [23]. A comparison between competing emission channels of excited $\mathrm{TaO}^{*}$ and $\mathrm{HoO}^{*}$ molecules revealed their dependence on the concentration of implanted Ho atoms, as follows: a higher concentration led to higher yields of the $\mathrm{HoO}^{*}$ emission, while a lower concentration produced higher yields of the $\mathrm{TaO}^{*}$ emission.

The temperature increase up to $1500 \mathrm{~K}$ stimulates the diffusion of oxygen from the bulk towards the surface while the thermal desorption of oxide molecules is still not effective at $T<1500 \mathrm{~K}$. Apparently, the growth rate of oxide concentration due to the diffusion is higher than its depletion due to both the thermal desorption and the ion bombardment, which makes formation of the $\mathrm{Ta}_{n} \mathrm{O}$ molecules more effective so that the yields of sputtered $\mathrm{Ta}_{n} \mathrm{O}^{+}$ions become enhanced. At $T>$ $1700 \mathrm{~K}$, oxygen is essentially cleaned off the surface, and the yield of the $\mathrm{Ta}_{n} \mathrm{O}^{+}$ions falls down to zero while the yields of $\mathrm{Ta}_{n}{ }^{+}$and $\mathrm{Ta}_{n-1} \mathrm{Nb}^{+}$ions from such clean surfaces are then stabilized reaching their maximums.

Compared with the $\mathrm{Au}^{-}$atomic ion bombardment, polyatomic $\mathrm{Au}_{3}{ }^{-}$projectiles appear to be more efficient to clean the surface from oxygen and other contaminants. This enhances the yield of $\mathrm{Ta}_{n}{ }^{+}$and $\mathrm{Ta}_{n-1} \mathrm{Nb}^{+}$ions so that the clusters with higher $n$ numbers can be observed in the mass spectra at lower temperature. At the same time, the polyatomic ion bombardment produces a higher concentration of implanted gold atoms. Under such conditions, not only atomic $\mathrm{Au}^{+}$ions can be observed (as was also under atomic ion bombardment) but also a variety of mixed cluster ions of $\mathrm{Ta}_{n-1} \mathrm{Au}^{+}$. At higher temperatures than $2100 \mathrm{~K}$, the equilibrium concentration of gold atoms on the surface apparently falls to zero too. Considering experimental findings and trying to compare the influence of surface conditions on the cluster ion yields, one should not forget that, compared with the atomic bombardment, the polyatomic bombardment can enhance the cluster component in secondary ion emission due to a differently developing ion sputtering process [6]. This can result in both better surface cleaning and the more intense cluster ion emission [10-12]. These two factors can be separated from each other only at high target temperatures when the surface is clean.

Unfortunately, it is currently impossible to quantitatively estimate weights of the different emission channels because 
ionization probabilities of many detected cluster ions are unknown, some of them may also depend on the target temperature. Nevertheless, the qualitative considerations given previously clearly indicate the redistribution of the sputtered substance between these channels, namely between emission of homogeneous and different heterogeneous (mixed) clusters.

The complexities described above have to be taken into account when temperature dependence of secondary ion emission is measured and interpreted.

\section{Acknowledgements}

The authors are grateful to the Fund for Scientific Research-Flanders, Brussels, Belgium and to the US Department of Energy, BES-Materials Sciences, which supported this work under Contract No.W-31-109-ENG-38. Special thank to Leonid Stroev and Yulia Belykh for the help in measurements.

\section{References}

[1] H.H. Andersen, Mat. Fys. Medd. Dan. Vid. Selsk. 43 (1993) 127.

[2] M.L. Yu, in: R. Behrisch, K. Wittmaack (Eds.), Sputtering by Particle Bombardment III, Springer, Berlin, 1991, p. 91.

[3] P. Sigmund, C. Claussen, J. Appl. Phys. (1981) 52.

[4] I.S. Bitensky, E.S. Parilis, Nucl. Instr. Meth. B 21 (1987) 26.

[5] H.M. Urbassek, Nucl. Instr. Meth. B 31 (1988) 79.
[6] S.F. Belykh, V.I. Matveev, I.V. Veryovkin, A. Adriaens, F. Adams, Nucl. Instr. Meth. B 155 (1999) 409.

[7] I.A. Wojciechowski, P. Bertrand, M.V. Medvedeva, V.K. Ferleger, Nucl. Instr. Meth. B 179 (2001).

[8] V.I. Matveev, Izv. Akad. Nauk Fiz. 66 (4) (2002) 516 (in Russian).

[9] R. Heinrich, C. Staudt, M. Wahl, A. Wucher, in: A. Benninghoven, P. Bertrand, H.N. Migeon, H.W. Werner (Eds.), Secondary Ion Mass Spectrometry. SIMS XII, Elsevier Science, Amsterdam, 2000, p. 111.

[10] S.F. Belykh, U.Kh. Rasulev, A.V. Samartsev, S.V. Verkhoturov, I.V. Veryovkin, Mikrochim. Acta 15 (Suppl.) (1998) 379.

[11] S.F. Belykh, U.Kh. Rasulev, A.V. Samartsev, I.V. Veryovkin, Nucl. Instr. Meth. B 136-138 (1998) 773.

[12] S.F. Belykh, B. Habets, U.Kh. Rasulev, A.V. Samartsev, L.V. Stroev, I.V. Veryovkin, Nucl. Instr. Meth. B 164-165 (2000) 809.

[13] S.F. Belykh, V.V. Palitsin, I.V. Veryovkin, A. Adriaens, F. Adams, Nucl. Instr. Meth. B 203 (2003) 164.

[14] A.V. Samartsev, A. Wucher, Appl. Surf. Sci., in press.

[15] C. Staudt, R. Heinrich, P. Mazarov, A. Wucher, T.I. Tugushev, N.Kh. Dzhemilev, Nucl. Instr. Meth. B 164-165 (2000) 715.

[16] S.F. Belykh, Yu.S. Belykh, A.V. Samartsev, L.V. Stroev, Proceedings of the XIV International Conference on Ion-Surface Interaction (ISI1999), Zvenigorod, Russia, Vol. 1, 1999, p. 256.

[17] S.F. Belykh, R.N. Evtukhov, J.N. Lysenko, U.Kh. Rasulev, Rev. Sci. Instr. 63 (1992) 2458

[18] T. Yano, T. Ooie, M. Yoneda, M. Katsumura, T. Hino, T. Araki, J. Jpn. Inst. Met. 61 (1997) 71.

[19] L. Malter, D.B. Lungmuir, Phys. Rev. 55 (1939) 743.

[20] V.N. Ageev, N.I. Ionov, Sov. Phys. Solid State 13 (1971) 1305.

[21] A.Y. Tontegode, F. ElBalghiti, M. Bacal, Plasma Source Sci. Technol. 5 (1996) 412

[22] N.R. Gall, E.V. Rut'kov, A.Ya. Tontegode, JETP 95 (2) (2002) 294.

[23] S.F. Belykh, R.N. Evtukhov, I.V. Redina, V.K. Ferleger, Nucl. Instr. Meth. B 95 (1995) 300. 